\title{
Il ricircolo nell'accesso vascolare per emodialisi
}

\author{
P. Calzavara \\ Reparto di Nefrologia e Dialisi - Ospedale Regionale di Treviso
}

$\mathbf{P}$

er ricircolo $(\mathrm{R})$ nell'accesso vascolare, in corso di trattamento emodialitico, s'intende il passaggio di sangue già dializzato dall'ago venoso a quello arterioso.

Il R può svilupparsi: 1) quando il flusso nel circuito extracorporeo supera quello ematico all'interno della fistola determinando un flusso retrogrado dall'ago venoso a quello arterioso; 2) quando è presente un'elevata resistenza al deflusso venoso; 3) quando l'ago arterioso e venoso sono troppo vicini o invertiti.

Il $R$ va accuratamente ricercato nei pazienti in cui, senza alcuna causa apparente, si osservi un incremento della concentrazione di BUN e/o di creatinina o si presentino dei sintomi di sottodialisi. Il $\mathrm{R}$ infatti riduce l'efficacia dialitica soprattutto delle piccole molecole. È stato riportato come, a fronte di $\mathrm{R}$ maggiori del $15 \%$, l'efficacia dialitica prescritta si riduca del $10 \%$ (1).

La formula seguente evidenzia la relazione tra $\mathrm{R}$ e clearance effettiva del filtro:

$$
\mathrm{Ke}=\mathrm{K}[1-\mathrm{R} / 1-\mathrm{R}(1-\mathrm{K} / \mathrm{Qb})](2)
$$

dove: $\mathrm{Ke}=$ clearance effettiva, $\mathrm{K}=$ clearance del filtro senza $\mathrm{R}, \mathrm{Qb}=$ flusso ematico.

L'entità del $\mathrm{R}$ aumenta con l'incrementarsi del Qb e soprattutto nelle dialisi ad elevati flussi, può verificarsi anche in assenza di trombosi, stenosi, calcificazioni o di altre alterazioni anatomomiche a carico dei vasi sanguigni, ma semplicemente per la presenza di vene arterializzate di piccolo calibro (3-5).

La percentuale di $\mathrm{R}$ viene calcolata attraverso la formula classica proposta da Gotch (6):

$$
\begin{aligned}
& \% \mathrm{R}=(\mathrm{C} \text { BUN s }-\mathrm{C} \text { BUN a }) / \\
& (\mathrm{C} \text { BUN s - C BUN v }) \times 100
\end{aligned}
$$

dove $\mathrm{C}=$ concentrazione plasmatica, $\mathrm{BUN}=$ azotemia, $\mathrm{s}=$ sangue sistemico (prelevato da una vena dell'arto controlaterale a quello della fistola artero-venosa), $a=$ sangue linea arteriosa, $v=$ sangue linea venosa.

In teoria il $\mathrm{R}$ è assente quando $\mathrm{C} B U N$ a $=\mathrm{C} B U N \mathrm{~s}$, mentre è presente quando $\mathrm{C}$ BUN a $<$ C BUN s.

Il prelievo dei tre campioni di sangue deve essere contemporaneo ed eseguito entro la prima ora di dialisi (4). Questo termine di tempo viene consigliato per ovviare alla vasocostrizione periferica che si può manifestare durante il trattamento extracorporeo. Tale vasocostrizione rallenterebbe la depurazione nell'arto opposto a quello della fistola artero-venosa portando ad una soprastima del R.

Dopo 5, 60, 120 minuti dall'inizio dell'emodialisi sono state riportate concen- trazioni di BUN più elevate, nell'arto opposto alla fistola, rispettivamente del 1026-36\% rispetto alle concentrazioni ottenute prelevando il sangue dalla linea arteriosa (squilibrio veno-venoso) (7).

Sarebbe più corretto, ma improponibile, prelevare da una arteria periferica invece che dalla vena dell'arto controlaterale a quello della fistola in quanto, durante l'emodialisi, la concentrazione di BUN nel sangue arterioso è lievemente inferiore a quella nel sangue venoso (squilibrio artero-venoso) per effetto del ricircolo cardiopolmonare (il ricircolo cardiopolmonare non esiste in caso si utilizzino cateteri per vene centrali) (8).

Il metodo classico, su riportato, soprastima secondo alcuni Autori del 7\% (9), secondo Altri del 12.5\% (10).

La difficoltà, a volte, a reperire una vena facilmente pungibile nell'arto controlaterale, il rischio di procurare ematomi pungendo un vaso in corso di eparinizzazione e la necessità di risparmiare vasi potenzialmente utili per successive fistole artero-venose hanno fatto sì che siano stati indagati altri metodi.

Kaufman et al (11) hanno proposto un sistema mediante il quale, raffreddando la linea venosa, il $\mathrm{R}$ viene misurato dal calo della temperatura nella linea arteriosa.

Greenwood et al (12) hanno studiato un metodo simile basato sull'infusione di un bolo di fisiologica a $4^{\circ} \mathrm{C}$ nella linea ve- 
nosa e rilevato, in caso di $\mathrm{R}$, nella linea arteriosa.

Hester et al (13) hanno calcolato il R dalla variazione della trasmissione di luce nella linea arteriosa, proporzionale all'ematocrito, infondendo una soluzione salina nella linea venosa.

Depner et al (14), infine, hanno impiegato un sensore ad ultrasuoni inserito a livello della linea arteriosa che misura il $R$ dall'eventuale variazione indotta dall'infusione di un bolo di soluzione fisiologica nella linea venosa.

Questi metodi però non sono sempre di facile applicazione per cui, routinariamente, si ricorre ancora ai metodi basati su prelievi ematici.

Oltre alla tecnica classica, basata su tre campioni di sangue, sono state proposte varie metodiche alternative che evitano il prelievo dall'arto controlaterale a quello della fistola artero-venosa sostituendolo, in vari modi, con un prelievo dalla linea arteriosa.

Un metodo, quello dello stop flusso, è di prelevare il campione dalla linea arteriosa dopo aver fermato la pompa-sangue per circa 1 minuto; un secondo prelievo dalla linea arteriosa ed un prelievo dalla linea venosa vengono eseguiti dopo aver fatto ripartire la pompa. Con questa procedura si può sottostimare il valore di $\mathrm{R}$ $\operatorname{del} 5 \%$ (15).

Un altro, quello a basso flusso, è di prelevare dopo 30 minuti di trattamento emodialitico dalla linea arteriosa e venosa e quindi ridurre immediatamente il $\mathrm{Qb}$ a $50 \mathrm{ml} / \mathrm{min}$ per eseguire, entro $30 \mathrm{se}$ condi, il prelievo dalla linea arteriosa. Gli Autori di questa tecnica riportano risultati sovrapponibili al sistema dello stop flusso (16).

Un altro metodo è quello di eseguire un prelievo dalla linea arteriosa previa occlusione di 1 minuto tra ago arterioso e venoso e poi, una volta tolta l'occlusione, procedere ai prelievi dalla linea arteriosa e dalla linea venosa. Questo metodo però può essere rischioso per il funzionamento della fistola stessa (5).

Un altro è quello di prelevare un campione di sangue dalla linea arteriosa subito all'inizio della dialisi ed eseguire i prelievi dalla arteria e dalla vena dopo 5, dall'attacco. Questo metodo sovrastima rispetto al metodo classico del 4\% (17). Un altro ancora è di prelevare un campione di sangue dalla linea arteriosa $15^{\text {, }}$ dopo il termine del trattamento dialitico. Gli Autori hanno anche confrontato tale procedura con il metodo classico, eseguito 5 minuti prima della fine del trattamento, evidenziando una buona correlazione tra le due procedure (18).

Il metodo della venopuntura nell'arto controlaterale a quello della fistola e quello dello stop flusso non differiscono tra loro in modo significativo se eseguiti a 30 e 60 minuti dall'inizio del trattamento, mentre danno dei valori significativamente diversi a 120 minuti con percentuali di $\mathrm{R}$ più elevate con il primo metodo rispetto al secondo. A 120 minuti entrambi i metodi danno valori più elevati probabilmente per il calo del volume ematico e la riduzione della gettata cardiaca associata a rimozione di liquidi (4). Recentemente è stato criticato il prelievo dalla vena periferica in quanto fonte di errori significativi ed è stato proposto in alternativa il prelievo dalla linea arteriosa 5 minuti dopo il rallentamento della pompa-sangue a $50 \mathrm{ml} / \mathrm{min}$ (19).

I vantaggi dei metodi a due aghi, rispetto alla procedura classica, possono essere così riassunti: - preservare i vasi dell'arto controlaterale a quello della fistola per eventuali futuri accesi vascolari, - evitare il rischio di sanguinamento (per effetto dell'eparina) dalla puntura della vena periferica, - arrecare minor disagio ai pazienti in quanto evitano una puntura, possibilità d'esecuzione anche nei soggetti con vene difficilmente pungibili.

Nonostante queste varie metodiche alternative proposte per la misura del $\mathrm{R}$, la tecnica a tre aghi è tuttora considerata il "gold standard" anche se nei trattamenti ad alta efficienza è stata suggerita la tecnica dello stop flusso per la maggior possibilità di condizioni di disequilibrio del BUN tra comparti diversi del corpo (1).

La percentuale di R oltre la quale il valore ottenuto è da ritenersi "patologico" non è univoca: $20 \%$ secondo Alcuni (20), $15 \%$ secondo Altri (4), 10\% secondo Altri (21) ancora.

Ovviamente qualsiasi tecnica di misurazione venga impiegata, allorquando si evidenzi un R "patologico", è doveroso procedere ad un approfondimento diagnostico mediante dupplex scanner o eco-color-Doppler e/o fistolografia o uno studio angiografico più completo dell' arto sede dell'accesso vascolare.

Va comunque detto che pur essendo la prova di $\mathrm{R}$ utile nel poter predire una trombosi emodinamicamente significativa, non è stata finora evidenziata una correlazione tra aumento del $\mathrm{R}$ e trombo- si dell'accesso vascolare (22).

Un R elevato, infatti, non vuol dire presenza certa di stenosi nella vena arterializzata. Alcuni Autori (23) hanno osservato, con il metodo classico, in 1 solo caso su 9 pazienti, tutti con $\mathrm{R}>20 \%$, una stenosi venosa significativa alla fistolografia.

L'aumento della pressione venosa registrato dai monitors di dialisi costituisce un valido indice predittivo di stenosi venosa solo a bassi flussi ematici (200-225 $\mathrm{ml} / \mathrm{min}$ ) (24).

Un ruolo di rilievo nel determinare il $\mathrm{R}$ è svolto dal calibro degli aghi utilizzati: è stato dimostrato, appunto, come incrementando il $\mathrm{Qb}$ aumenti il R soprattutto con aghi di grosso calibro (5). Nello studio in questione il $\mathrm{R}$ è del $14.7 \%$ e del $14.3 \%$ rispettivamente aumentando il $\mathrm{Qb}$ da 250 a $300 \mathrm{ml} / \mathrm{min}$ usando aghi da 16 gauge, mentre aumenta da $19.6 \%$ al $21.9 \%$ incrementando il Qb da 350 a 400 $\mathrm{ml} / \mathrm{min}$ usando aghi da 14 gauge.

È noto come il $\mathrm{R}$ sia più frequente quando si dializza con monoago o con catetere monolume o bilume rispetto a quando si dializza con due aghi.

Il $\mathrm{R}$ può diventare particolarmente elevato in corso di dialisi mono-ago quando il $\mathrm{Qb}$ è basso e il ciclo aspirazione-restituzione di sangue è breve.

Con cateteri femorali a doppio lume è stata evidenziata l'importanza della lunghezza del catetere nel condizionare il $\mathrm{R}$. Con cateteri di $24 \mathrm{~cm}$ la percentuale di $\mathrm{R}$ è attorno al $10 \%$ sia a $\mathrm{Qb}$ di 250 che a Qb di $400 \mathrm{ml} / \mathrm{min}$, mentre con cateteri più corti di $15 \mathrm{~cm}$ la percentuale di $\mathrm{R}$ sale al 18 e $38 \%$ rispettivamente (25).

Concludendo il $\mathrm{R}$ è un test molto utile per il monitoraggio degli accessi vascolari rappresentando il mezzo più economico e a minor rischio per lo screening di stenosi dell'accesso vascolare.

Tuttavia, dalla revisione della letteratura, emerge come non esista ancora un accordo sulla tecnica da utilizzare per la misura del $\mathrm{R}$ e nemmeno sul valore soglia da considerare. È perciò auspicabile uno studio di confronto tra le varie procedure su un'ampia casistica, comparando i risultati ottenuti con quelli delle fistolografie, al fine di stabilire non solo la metodica più accurata, ma anche la percentuale oltre la quale il $\mathrm{R}$ è da considerarsi "patologico". 
BIBLIOGRAFIA

1. Ronco C. Dialysis delivery versus dialysis prescription. Int $\mathbf{J}$ Artif Organs 1993; 16: 628-35.

2. Gotch FA. Models to predict recirculation and its effect on treatment time in single-needle dialysis. In: Ringoir S, Vanholder R, Ivanovich $\mathrm{P}$, eds. First International Symposium on single-needle dialysis. Cleveland, OH, ISAO, 1984; 47-51.

3. Sherman RA, Levy S. Rate-related recirculation: effect of altering blood flow on dialysis recirculation. Am J Kid Dis 1991; 17: 170-4.

4. Collins DM, Lambert MB, Middleton JP, et al. Fistula disfunction: effect on rapid hemodialysis. Kidney Int 1992; 41: 1292-6.

5. Hasbargen JA, Weaver DT, Hasbargen BJ. The effect of needle gauge on recirculation, venous pressure and bleeding from puncture sites. Clin Nephrol 1995; 44: 322-4.

$6 . \quad$ Gotch FA. Hemodialysis: technical and kinetic considerations. In: Brenner BM and Rector FC, eds. The Kidney. WB Saunders, Philadelphia, 1976; 1672-704.

7. Depner TA, Rizwan S, Cheer AY, Wagner JM, Eder LA. High venous urea concentrations in the opposite arm. A consequence of hemodialysis-induced compartment disequilibrium. ASAIO Trans 1991; 37: M141-3.

8. Van Stone JC, Daugirdas JT. Principi di fisiologia. In: Dialisi. Centro Scientifico Editore, Torino 1995; 13-32.

9. Sherman RA. Recirculation revisited. Semin Dial 1991; 4: 221-3.

10. Tattersall JE, Farrington K, Raniga PD, et al. Haemodialysis recirculation detected by three-sample method is an artefact. Nephrol Dial Transplant 1993; 8: 60-3.

11. Kaufman AM, Draemer M, Godmere RO, et al. Hemodialysis access recirculation measurement by blood temperature monitoring - a new technique. J Am Soc Nephrol 1991; 2: 332 (abstr).

12. Greenwood RN, Aldridge C, Goldstein L, Baker LRI, Cattell WR. Assessment of arteriovenous fistulae from pressure and thermal dilution studies: clinical experience in forearm fistulae. Clin Nephrol 1985; 23: 189-97.

13. Hester RL, Ashcraft D, Curry E, Bower J. Non-invasive determination of recirculation in the patient on dialysis. ASAIO J 1992; 38: M190-3.

14. Depner TA, Krivitski NM, MacGibbon D. Hemodialysis access recirculation measured by ultrasound dilution. ASAIO J 1995; 41: M749-53.

15. Collins AJ. Applicazione clinica dell'emodialisi ad alta efficienza. In: Terapia dialitica (II ed). Hanley \& Belfus, Philadelphia 1994; 139 42.

16. Sherman RA. The measurement of dialysis access recirculation. Am J Kidney Dis 1993; 22: 616-21.

17. Kobrin SM, Kriger FL, Raja RM. Measurement of hemodialysis access recirculation: a two-needle method at the start of dialysis. ASAIO Trans 1989; 35: 508-10.

18. Pederson JA, Dunlay R, Williams C, Llach F. Two-needle calculation of recirculation compared with the standard three-needle method. Clin Nephrol 1990; 33: 203-6.

19. Van Stone JC. Peripheral venous blood is not the appropriate specimen to determine the amount of recirculation during hemodialysis. ASAIO Journal 1996; 42: 41-5.

20. Seidman MS, Lundis AP, Brown CD. Extent of blood recirculation during two-needle hemodialysis. Am Soc Artif Intern Organs 1979; 8: 56 (abstr).

21. Nardi L, Bosch J. Recirculation: review, techniques for measurement and ability to predict he- moaccess stenosis before and after angioplasty. Blood Purif 1988; 6: 85-9.

22. Gessaroli M. Utilizzazione degli accessi vascolari. Follow-up. In: Accessi vascolari per emodialisi. Ed. Minerva Medica, Torino 1994; 120-6.

23. Baldrati L, Neri L, Docci D, Pasini A, Capponcini C, Feletti C. La misurazione del ricircolo nelle fistole artero-venose. Giorn It Nefrol 1995; 12: 173-7.

24. Schwab SJ, Raymond J, Saeed M, Newman G, Dennis P, Bollinger R. Prevention of hemodialysis fistula thrombosis: elective detection and treatment of venous stenosis. Kidney Int 1989; 36: 707-11.

25. Kelber J, Delmez JA, Windus DW. Factors affecting delivery of high efficiency dialysis using temporary vascular access. Am J Kidney Dis 1993; 22: 24-9. 